Lüneburger Schriften zum Wirtschaftsrecht

\title{
Jennifer Pollex
}

Right on Course or Stranded?

An Analysis of the Equator Principles' Effectiveness 
Lüneburger Schriften zum Wirtschaftsrecht herausgegeben von Prof. Dr. jur. Dr. h.c. (GTU Tiflis) Thomas Schomerus

Band 24 


\section{Jennifer Pollex}

\section{Right on Course or Stranded?}

An Analysis of the Equator Principles' Effectiveness

\section{Nomos}


Die Deutsche Nationalbibliothek verzeichnet diese Publikation in der Deutschen Nationalbibliografie; detaillierte bibliografische Daten sind im Internet über http://dnb.d-nb.de abrufbar.

Die Deutsche Nationalbibliothek lists this publication in the Deutsche Nationalbibliografie; detailed bibliographic data is available in the Internet at http://dnb.d-nb.de.

ISBN 978-3-8329-7473-2

1. Auflage 2012

( ) Nomos Verlagsgesellschaft, Baden-Baden 2012. Printed in Germany. Alle Rechte, auch die des Nachdrucks von Auszügen, der fotomechanischen Wiedergabe und der Übersetzung, vorbehalten. Gedruckt auf alterungsbeständigem Papier.

This work is subject to copyright. All rights are reserved, whether the whole or part of the material is concerned, specifically those of translation, reprinting, re-use of illustrations, broadcasting, reproduction by photocopying machine or similar means, and storage in data banks. Under $\$ 54$ of the German Copyright Law where copies are made for other than private use a fee is payable to "Verwertungsgesellschaft Wort", Munich. 
Als Professor am Institut für Wirtschaftsrecht der Leuphana Universität Lüneburg hatte ich die große Freude, die Erstbetreuung der Diplomarbeit von Jennifer Pollex übernehmen zu dürfen. Mit ihrer Arbeit zu den Equator Principles (oder auf deutsch: Äquator-prinzipien) als mögliches Instrument des Umweltschutzes hat Frau Pollex Maßstäbe gesetzt, die weit über das hinausgehen, was im Rahmen einer wirtschaftsrechtlichen Diplomarbeit üblicherweise erwartet werden kann. Die Bedeutung des Bankensektors für den Umweltschutz und darüber hinausgehend die nachhaltige Entwicklung kann gar nicht hoch genug eingeschätzt werden. Zwar handelt es sich um ein auf Freiwilligkeit basierendes Prüfsystem für die Kredit-Finanzierung von Projekten. Jedoch sind die Prinzipien von fast 70 großen Banken aus 26 Staaten akzeptiert worden, und mehr als Zwei Drittel der Projektfinanzierungen für die Schwellenländer unterfielen den Regeln der Equator Principles. Allein dies macht sie zu einem weltweit bedeutenden, dabei aber im Bereich des Wirtschaftsrechts noch zu wenig beachteten Instrument.

Dies gilt vor allem auch vor dem Hintergrund der globalen Finanz- und Bankenkrise. Versuchungen, Umwelt- und Nachhaltigkeitsziele bei der Kreditvergabe hintenan zu stellen, hat der Bankensektor jedenfalls zum großen Teil widerstanden. Die Principles wurden nicht aufgeweicht, sondern eher noch verschärft. Insoweit gebührt der Verfasserin das Verdienst, das Werk, das ursprünglich 2009 als Diplomarbeit im Institut für Wirtschaftsrecht eingereicht worden war, im Hinblick auf die Finanzkrise auf Stand von 2011 gebracht zu haben. Die Leserinnen und Leser erhalten daher die Möglichkeit, sich in einem aktuellem, im Übrigen auch sprachlich sehr lesenswerten Werk grundlegend über die Möglichkeiten der Equator Principles im Hinblick auf den Umweltschutz zu informieren. Ich wünsche dem Buch daher eine möglichst große, auch internationale Verbreitung.

Lüneburg, im Januar 2012

Prof. Dr. Thomas Schomerus 


\section{Table of Contents}

List of Abbreviations

Introduction

Part A - I. Hopes and Expectations from Different Perspectives

1. Banks' Expectations

$\begin{array}{ll}1.1 \text { Reputation / Risk Management } & 17\end{array}$

1.2 Good Corporate Governance 18

1.3 Level Playing Field 18

1.4 Benefit of Voluntary Agreements 19

1.5 Reduced Cost of Capital Reserved for Loan Default 19

1.6 CEO Commitment 20

1.7 Business Considerations 20

2. NGO Expectations $\quad 20$

3. Sponsors' Expectations 21

4. Government Expectations 22

Part A - II. Content \& Implications of the Equator Principles 24

1. Preamble 24

2. Scope 25

3. Principle 1

4. Principle $2 \quad 28$

5. Principle $3 \quad 28$

6. Principles $4,5 \& 6$

7. Principles 7, 8 \& 9

8. Principle $10 \quad 31$

9. Conclusion $\quad 32$

Part B - I. Content Parameters 33

1. Objectives $\quad 33$

2. Responsibilities 36

3. Wording $\quad 37$

4. Flexibility vs. Rigidity 39

$\begin{array}{ll}\text { 5. Conclusion } & 40\end{array}$ 
1. Negotiation $\quad 41$

2. Monitoring \& Disclosure $\quad 42$

3. Consultation \& Grievance Mechanism 45

4. Evaluation, Revision \& Adaptation 46

5. Conclusion $\quad 48$

Part B - III. Adopting Institutions 49

1. Interest Homogeneity 49

2. Admission into the Circle of EPFIs 49

3. Power of Influence $\quad 50$

4. Free Riders and Competition $\quad 51$

5. Conclusion $\quad 52$

Part B - IV. Policy Style $\quad 54$

1. Political Context $\quad 54$

2. Roles of Government $\quad 55$

3. Alternative Instruments \& Policy Package $\quad 56$

4. Conclusion $\quad 57$

Part B - V. Consequences of Non-Compliance $\quad 58$

1. Liability Risks $\quad 58$

2. Internal Disciplinary Measures $\quad 60$

3. Other Financial Consequences 61

4. Case Study - BTC Pipeline Project 62

4.1 Project Facts 63

4.2 Allegations of Non-Compliance $\quad 63$

4.3 Banks' Reactions 64

4.4 Consequences of Non-Compliance $\quad 65$

4.5 Bottom Line 66

5. Conclusion 67

$\begin{array}{lr}\text { Part B - VI. Resources } & 68\end{array}$

1. Knowledge $\quad 68$

2. Staff and Independent Experts $\quad 69$

3. Motivation $\quad 70$ 
Part C - The Impact of the Equator Principles

1. Success and Failure in the Words of Scholars, Banks, NGOs \& Industry Consultants

2. Banks' Statistics of Rejected Projects

3. Extended Application and Virtuous Circle 74

4. Raised Awareness 75

5. Measuring the Principles' Impact 76

Taking Soundings: Conclusion and Outlook

Excursus: Impact of the Global Financial Crisis on the Equator Principles

Introduction

1. Background on the Global Financial Crisis $\quad 80$

2. EPFI Reporting $\quad 85$

3. ABN AMRO 85

5. Barclays $\quad 86$

6. WestLB 87

7. Citigroup $\quad 89$

8. Reforming CSR $\quad 89$

9. Equator Principles Strategic Review Process 91

10. Conclusions for the Equator Principles 93

11. Innovative and Scalable Solutions 93

12. Full Commitment and Transparency 95

13. Harmonious Decision-making 97

14. Conservation of Resources 98

15. Conclusion 98

$\begin{array}{ll}\text { Bibliography } & 100\end{array}$

$\begin{array}{ll}\text { Online Resources } & 105\end{array}$

$\begin{array}{ll}\text { Legal Sources } & 108\end{array}$ 


\section{List of Abbreviations}

\begin{tabular}{|c|c|}
\hline ABN AMRO & Algemene Bank Nederland-Amsterdam Roterdam \\
\hline AP & Action Plan \\
\hline Art. & Article \\
\hline Aufl. & Auflage \\
\hline $\mathrm{Bd}$. & Band \\
\hline $\mathrm{BP}$ & British Petrol \\
\hline BTC pipeline & Baku-Tbilisi-Ceyhan Pipeline \\
\hline CEO & Chief Executive Officer \\
\hline CSR & Corporate Social Responsibility \\
\hline Dr. & Doctor \\
\hline EEA & European Environmental Agency \\
\hline EHS Guidelines & Environmental, Health and Safety Guidelines \\
\hline EIA & Environmental Impact Assessment \\
\hline EP & Equator Principles \\
\hline EPFI & Equator Principles Financial Institution(s) \\
\hline Etc. & et cetera \\
\hline EU & European Union \\
\hline EUR & Euro \\
\hline f. & et sequens \\
\hline FAQ & Frequently Asked Questions \\
\hline ff. & et sequentes \\
\hline $\mathrm{FH}$ & Fachhochschule \\
\hline HSBC & Hongkong and Shanghai Banking Corporation \\
\hline http & hypertext transfer protocol \\
\hline ibid. & Ibidem \\
\hline I.e. & Id est \\
\hline IFC & International Finance Corporation \\
\hline Matr. & Matrikel \\
\hline IRB & Internal Ratings-Based \\
\hline NGO & Non-Government Organisation(s) \\
\hline OECD & Organisation for Economic Co-operation and Development \\
\hline Para. & Paragraph \\
\hline PR & Public Relations \\
\hline Prof. & Professor \\
\hline Rn. & Randnummer \\
\hline p. & Page \\
\hline PKK & Partiya Karkerên Kurdistan \\
\hline
\end{tabular}


